particularly relevant to auto-Ags that consist of DNA- or RNA-containing immune complexes. Although $\mathrm{Ab}$ responses to some Ags moderately increased following STAL treatment, $100 \%$ effective depletion of Ag-specific $\mathrm{B}$ cells may not be necessary, as a reduction (vs. abolishment) in harmful auto-Abs may be sufficient for clinical benefit. Considering that most autoimmune disorders also contain a significant $\mathrm{T}$ cell component, it may be necessary to combine STAL treatment with additional therapies aimed at inhibiting $\mathrm{T}$ cell responses such as costimulatory blockade or Ag targeting to pDCs. Despite these questions, the new platform introduced by Macauley and colleagues opens an exciting avenue for $\mathrm{B}$ cell manipulation, and adds to an ever-growing body of work that seeks to manipulate Ag-specific immune responses.

\section{Acknowledgments}

We thank Christiane Dresch for critical comments. Our research described here was supported by NIH grants R01-AI52203 and R37-AAI4257 to E.A. Clark.

Address correspondence to: Edward A. Clark, Department of Immunology, Uni- versity of Washington, Office I-319A, HSC, Box 357242, 1959 NE Pacific St. NE, Seattle, Washington 98195, USA. Phone: 206.543.8706; Fax: 206.616.6772; E-mail: eaclark@uw.edu.

1. Caminschi I, Lahoud MH, Shortman K. Enhancing immune responses by targeting antigen to DC. Eur J Immunol. 2009;39(4):931-938.

2. Macauley MS, et al. Antigenic liposomes displaying CD22 ligands induce antigen-specific B cell apoptosis. J Clin Invest. 2013;123(7):3074-3083.

3. Otipoby KL, Draves KE, Clark EA. CD22 regulates $\mathrm{B}$ cell receptor-mediated signals via two domains that independently recruit Grb2 and SHP-1.J Biol Chem. 2001;276(47):44315-44322.

4. Nitschke L. CD22 and Siglec-G: B-cell inhibitory receptors with distinct functions. Immunol Rev. 2009;230(1):128-143.

5. Burgering BM, Medema RH. Decisions on life and death: FOXO Forkhead transcription factors are in command when $\mathrm{PKB} /$ Akt is off duty. J Leukoc Biol. 2003;73(6):689-701.

6. Denis O, Latinne D, Nisol F, Bazin H. Resting B cells can act as antigen presenting cells in vivo and induce antibody responses. Int Immunol. 1993;5(1):71-78.

7. Kawamura H, Berzofsky JA. Enhancement of antigenic potency in vitro and immunogenicity in vivo by coupling the antigen to anti-immunoglobulin. Immunol. 1986;136(1):58-65.

8. Chaplin JW, Kasahara S, Clark EA, Ledbetter JA. Anti-CD180 (RP105) activates B cells to rapidly produce polyclonal Ig via a T cell and MyD88-independent pathway. J Immunol. 2011;187(8):4199-4209.

9. Pulendran B, Tang H, Denning TL. Division of labor, plasticity, and crosstalk between dendritic cell subsets. Curr Opin Immunol. 2008;20(1):61-67.

10. Dudziak D, et al. Differential antigen processing by dendritic cell subsets in vivo. Science. 2007; 315(5808):107-111

11. Chappell CP, Draves KE, Giltiay NV, Clark EA. Extrafollicular B cell activation by marginal zone dendritic cells drives $\mathrm{T}$ cell-dependent antibody responses. J Exp Med. 2012;209(10):1825-1840.

12. Corbett AJ, et al. Antigen delivery via two molecules on the CD8- dendritic cell subset induces humoral immunity in the absence of conventional "danger". Eur I Immunol. 2005;35(10):2815-2825.

13. Loschko J, et al. Antigen targeting to plasmacytoid dendritic cells via Siglec-H inhibits Th cell-dependent autoimmunity. IImmunol. 2011;187(12):6346-6356.

14. Lahoud MH, et al. The C-type lectin Clec12A present on mouse and human dendritic cells can serve as a target for antigen delivery and enhancement of antibody responses. J Immunol. 2009;182(12):7587-7594.

15. Fanning SL, et al. Receptor cross-linking on human plasmacytoid dendritic cells leads to the regulation of IFN-alpha production. J Immunol. 2006; 177(9):5829-5839.

16. Rock J, et al. CD303 (BDCA-2) signals in plasmacytoid dendritic cells via a BCR-like signalosome involving Syk, Slp65 and PLCgamma2. Eur J Immunol. 2007;37(12):3564-3575

17. Ehrenforth S, et al. Incidence of development of factor VIII and factor IX inhibitors in haemophiliacs. Lancet. 1992;339(8793):594-598.

18. Stonebraker JS, Bolton-Maggs PH, Michael Soucie J, Walker I, Brooker M. A study of variations in the reported haemophilia $B$ prevalence around the world. Haemophilia. 2010;18(3):e91-e94.

19. Dimichele DM. Management of factor VIII inhibitors. Int J Hematol. 2006;83(2):119-125.

\title{
Linking MLL and the HGF-MET signaling pathway in liver cancer
}

\author{
Jens U. Marquardt'1,2 and Snorri S. Thorgeirsson'1
}

\begin{abstract}
${ }^{1}$ Laboratory of Experimental Carcinogenesis (LEC), Center for Cancer Research, National Cancer Institute, NIH, Bethesda, Maryland, USA
\end{abstract} 2Department of Medicine I, Johannes Gutenberg University, Mainz, Germany.

\begin{abstract}
Mixed-lineage leukemia (MLL; also known as myeloid/lymphoid), the human homolog of trithorax in Drosophila, is a transcriptional coactivator that plays an essential role during early development and hematopoiesis. Furthermore, MLL is critically involved in the epigenetic regulation of cell cycle, senescence, DNA damage, and stem cell self-renewal. Chromosomal aberrations of $M L L$ in acute leukemias are well documented, but the role of this gene in solid malignancies remains unclear. In this issue of the JCI, Takeda et al. describe a novel epigenetic link between MLL and the HGFMET signaling pathway conferring invasive and metastatic properties to hepatocellular carcinoma cells.
\end{abstract}

The genomic landscapes of most common forms of human cancer have now been defined (1). One of the most interesting findings emerging from these studies

Conflict of interest: The authors have declared that no conflict of interest exists.

Citation for this article: J Clin Invest. 2013;

123(7):2780-2783. doi:10.1172/JCI70235. was the identification of approximately 140 genes that, when altered by intragenic mutations, can promote or "drive" tumorigenesis in human cancers. Importantly, these driver genes can be classified into one or more of 12 signaling pathways regulating three fundamental cellular processes: cell fate, cell survival, and genome maintenance (1). It is self-evident that better understanding and therapeutic use of these signaling pathways promises to improve both treatment and prevention of human cancer.

Among the druggable driver genes are several key oncogenic molecules, such as EGFR, BRAF, and MET, motivating the development of targeted agents that have already improved the outcome of the subgroups of patients with malignancies harboring activating mutations in these genes. However, in hepatocellular carcinomas (HCCs), no such clear "oncogene addiction” exists, highlighting a role for alternative modes of oncogene activation, such as by epi-driver genes, which are epigenetically altered to be aberrantly expressed in cancer, conferring a growth advantage $(1,2)$. HCC most often develops in the context of a chronically inflamed microenviron- 


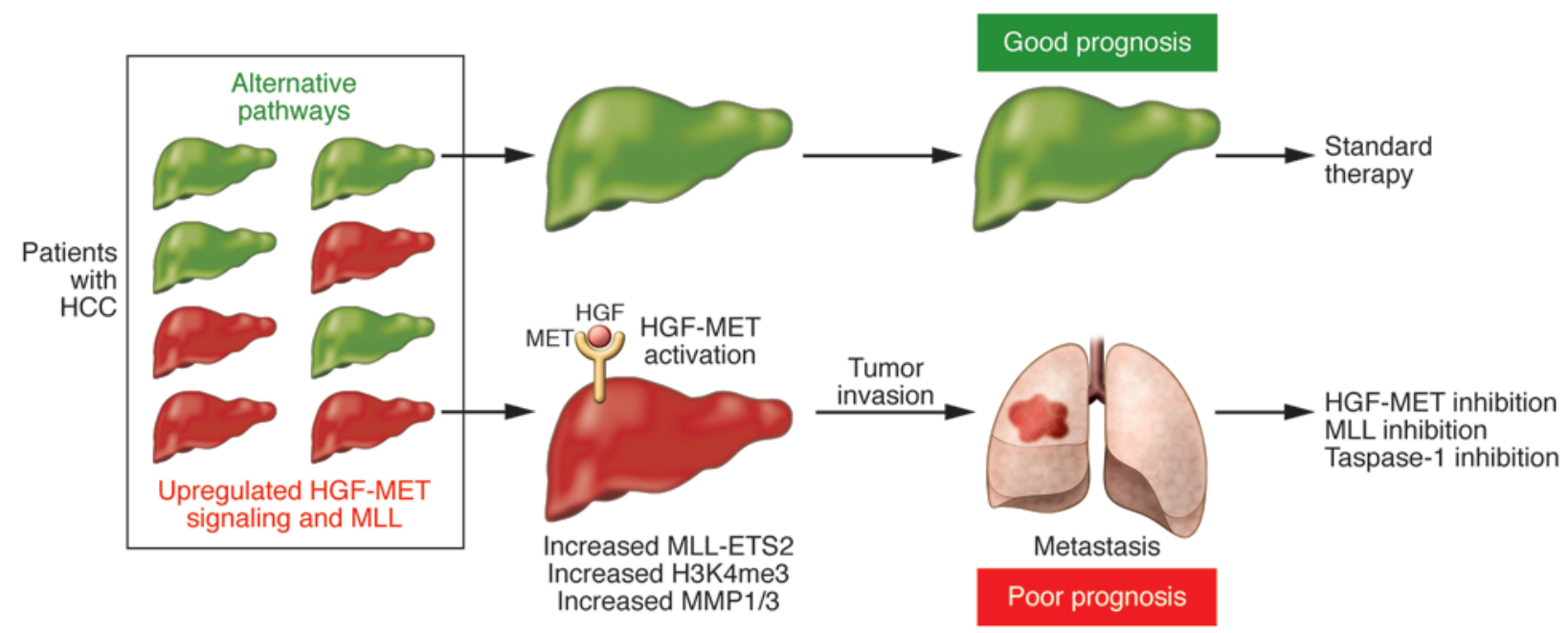

\section{Figure 1}

Clinical impact of MLL activation in human liver cancer. HCC is a molecularly heterogeneous disease. The present study by Takeda et al. (4) indicates that a subclass of patients with activated HGF-MET signaling might also display induction of MLL expression. This induction stabilizes the MLL-ETS2 complex and increases H3K4me3. Activation of this signaling cascade confers invasive tumor growth of HCC cells and seeding of distant metastasis via MMP1 and MMP3 upregulation, which might explain the poor outcome associated with MET activation. Therefore, this newly described engagement of HGF-MET and MLL might provide a promising approach for selection of HCC patients most likely to benefit from targeted therapies with MLL, taspase-1, or MET inhibitors and ultimately improve patient survival.

ment, resulting in extraordinary phenotypic heterogeneity and dismal prognosis of the affected patients (3). The underlying genetic and epigenetic complexity is underappreciated by current histological classifications and imaging techniques. Therefore, selection of patients most likely to benefit from a specific therapy is a major challenge for translational efforts in HCC. Here, we summarize the role of activated HGF-MET signaling in HCC and delineate the mechanistic implications of the study in this issue by Takeda et al. (4), which describes an epigenetic link between mixed-lineage leukemia (MLL) and the HGF-MET signaling pathway supporting invasion and metastasis in liver cancer. We also highlight the importance of this novel cooperation for therapeutic approaches in HCC.

\section{MLL in development and disease}

The MLL protein and its Drosophila homo$\log$, trithorax, are essential regulators of development, required for proper homeotic gene expression. MLL exerts its action in part by encoding a DNA-binding protein that methylates histone $\mathrm{H} 3$ lysine 4 (H3K4) after proteolytic cleavage by taspase- 1 (encoded by TASP1), thereby regulating expression of targets that include multiple Hox genes (5). Disruption of MLL is associated with Wiedemann-Steiner Syndrome (characterized by hairy elbows, short stature, facial dysmorphism, and developmen- tal delay; OMIM 605130), and constitutive loss of Mll leads to embryonic lethality in mice (6). The affected animals show considerable growth retardation, hematopoietic abnormalities, bidirectional homeotic transformation of the axial skeleton, and sternal malformations, indicating the importance of MLL in a variety of developmental processes. Furthermore, conditional disruption of $M l l$ in hematopoietic cells leads to reduced self-renewal of hematopoietic stem cells (7). More importantly, genetic alterations of $M L L$, in particular translocations, are frequently observed in acute leukemias and are associated with a poor prognosis (8). In most cases, protumorigenic activity of MLL is caused by loss of H3K4 methyltransferase activity. The critical role of MLL for hematopoietic stem cells may further explain the diverse oncophenotypes associated with MLL disruption, such as acute myeloid leukemia (AML), acute lymphoblastic leukemia (ALL), or mixed-lineage (biphenotypic) leukemia (MLL). Another interesting finding is the association of MLL with therapyrelated leukemias that develop on the basis of previous treatment with a topoisomerase II inhibitor in other malignancies, suggesting a role for MLL in carcinogenic genetic recombination under selective pressure (9). In addition to the activation of Hox genes, MLL signaling leads to downstream activation of other key oncogenic factors, such as EPHA7, MEIS1, and FLT3, as well as aberrant expression of GSK-3, HSP-90, ERK, and RAS pathways (10). The role of MLL in carcinogenesis beyond the hematopoietic system is largely unknown.

\section{Role of HGF-MET signaling in liver physiology and disease}

Despite the molecular and phenotypic heterogeneity of HCCs, several genetic and epigenetic alterations in key molecules and signaling pathways have been identified that promote hepatocarcinogenesis (2). Among the most prominent is the HGF-MET pathway. HGF and its receptor, c-MET, are crucial for liver homeostasis, and the pathway is activated during virtually all acute as well as chronic liver pathologies (11). HGF-MET signaling is essential for both hepatocyte- and stem cell-driven liver regeneration and functions through versatile interaction with a plethora of downstream signaling molecules $(12,13)$. Most importantly, high HGF-MET levels lead to activation of stress- and survivalassociated molecules, including MAPK, ERK, JNKs, PI3K-AKT, and STATs (14). Additionally, tight interaction with NF-кB upon liver damage is observed $(15,16)$. More recently, it has been shown that MET acts as a proto-oncogene in several tumors by responding to pro-oncogenic stimuli of altered tissue microenvironments frequently observed in the liver (e.g., in response 
to inflammation, injury, or hypoxia) and activates invasive and metastatic programs (17). Activated MET is significantly associated with vascular invasion, neoangiogenesis, and poor patient outcome in liver cancer (18). Given the importance of MET for hepatic stem cells, a progenitor cell feature is frequently associated with MET activation in HCC, making the pathway an attractive target for therapeutic interventions. Results from two recently concluded clinical trials further indicate that inhibition of the pathway might provide an effective and safe second-line option for patients with advanced HCCs and activated MET signaling $(19,20)$.

\section{Epigenetic convergence of MLL with HGF-MET signaling}

Our understanding of the genetic and epigenetic landscape of HCC is still in its infancy. Therefore, the discovery of novel molecules leading to activation of key signaling pathways is of major importance for predicting individual susceptibility and for identifying patients most likely to benefit from a specific therapy. Takeda et al. now demonstrate that MLL plays an intriguing and unexpected role in hepatocarcinogenesis by promoting the invasive capability of HGF-MET signaling (4). The authors elegantly show that MLL-dependent epigenetic activation of MMP1 and MMP3 via $\mathrm{H} 3 \mathrm{~K} 4$ trimethylation $(\mathrm{H} 3 \mathrm{~K} 4 \mathrm{me} 3)$ is required for the HGF-MET-induced invasive capacity of HCC cell lines. To circumvent embryonic mortality of MLL deletion, the authors used a taspase-1-noncleavable hypomorphic variant of $M l l$ that displayed a similar phenotype to the previously generated Tasp $1^{-/-}$mouse (21). Interestingly, aside from the expected homeotic defects in the axial skeleton, genetic loss of $\mathrm{Mll}$ also led to defective neurite outgrowth and myoblast migration. This phenotype was previously shown to result from HGF and MET deficiency, but it is not seen in Hox-knockout mice, suggesting a Hox-independent role of HGF-MET signaling (22). The high phenotypic overlap with defective migratory capacity (i.e., neurite outgrowth and myoblast migration) among $\mathrm{Mll}^{-/-}$, Tasp $1^{-/-}$, and $\mathrm{Hgf}^{/-} \mathrm{Met}^{-1-}$ mice led the authors to further investigate the role of MLL as a critical downstream component of HGF-MET in HCC cells. Consistently, Takeda et al. found that RNAi-mediated knockdown of MLL had no effect on MET expression or cell proliferation, but significantly affected the invasive properties in HepG2 and HLE cells.
Comparative analysis of HGF-MET signaling in the HCC cells as well as the mouse model convincingly showed that the link between MLL and invasiveness was conferred by direct transcriptional activation of $M M P 1$ and $M M P 3$. The authors further demonstrated that HGF-MET-induced stabilization of the MLL-ETS2 complex is responsible for $\mathrm{H} 3 \mathrm{~K} 4 \mathrm{me} 3$ in a Hoxindependent manner. Finally, the authors validated the critical role of this new mechanistic link by performing xenotransplantation assays in highly immunocompromised NOD-SCID $\mathrm{Il}_{2 \mathrm{rg}^{--}}$mice in vivo. In agreement with the above findings, RNAimediated knockdown of MLL in HepG2 cells significantly decreased the incidence of metastasis from $86 \%$ to $25 \%$ (4), which suggests that MLL plays a key role in invasion of liver cancer cells and highlights the molecule as a potential target for therapeutic interventions.

\section{Concluding remarks and future directions}

The conceptually novel and mechanistically well-performed study by Takeda and colleagues (4) reveals a previously unrecognized mode for HGF-MET-induced activation of invasive properties in liver and potentially other cancer types (Figure 1). Given the prognostic role of activated MET signaling in HCC and the success of recent clinical trials using MET inhibitors, it will now be crucial to investigate whether activated MET signaling is required for the established concerted action of HGFMET and MLL, or if activation of MLL can induce invasion of HCC cells independent of HGF-MET signaling, e.g., by genetic alterations of $M L L$ during hepatocarcinogenesis $(19,20)$. This is particularly important for clinical translation of the findings, since recent studies using next-generation sequencing approaches demonstrated that chromatin regulators, including MLL, are mutated in a substantial proportion of HCCs (23). If MET-dependent activation can be validated, it will also be essential to determine whether MLL activation can drive the development of metastasis. In this case, a routine screen for MLL activation in MET-overexpressing tumors and subsequent MLL- or taspase-1-based treatment strategies might be warranted. If genetic aberration of $M L L$ leads to autonomous regulation, screening of MLL would be necessary to predict the benefit of MET-based treatment modalities, similar to KRAS status in other cancers (24). Given the abundance of malignancy-related genetic alterations in molecules critical for chromatin remodeling (e.g., ARID1A, ARID1B, ARID2, and MLL3), the importance and potential redundancies of epigenetic modifiers in the regulation of invasion should be tested (25). Finally, the present study convincingly demonstrates the cooperation of HGF-MET and MLL engagement in invasion. However, while therapeutic targeting of this interaction might reduce the incidence of distant metastasis, it might not affect the proliferative capacity of the original tumor. Notably, it has been repeatedly demonstrated that control of the intrahepatic tumor load is an essential determinant of patient outcome, so the prognostic relevance of HGF-METinduced MLL activation still remains to be determined (26).

\section{Acknowledgments}

J.U. Marquardt is supported by grant MA 4443/2-1 from the German Research Foundation.

Address correspondence to: Snorri S. Thorgeirsson, Laboratory of Experimental Carcinogenesis, Center for Cancer Research, National Cancer Institute, NIH, 37 Convent Drive, Room 4146, Bethesda, Maryland 20892, USA. Phone: 301.496.1935; Fax: 301.496.0734; E-mail: snorri_thorgeirsson@nih.gov.

1. Vogelstein B, Papadopoulos N, Velculescu VE, Zhou S, Diaz LA Jr, Kinzler KW. Cancer genome landscapes. Science. 2013;339(6127):1546-1558.

2. Marquardt JU, Galle PR, Teufel A. Molecular diagnosis and therapy of hepatocellular carcinoma (HCC): an emerging field for advanced technologies. J Hepatol. 2012;56(1):267-275.

3. Farazi PA, DePinho RA. Hepatocellular carcinoma pathogenesis: from genes to environment. Nat Rev Cancer. 2006;6(9):674-687.

4. Takeda $S$, et al. HGF-MET signals via the MLLETS2 complex in hepatocellular carcinoma. J Clin Invest. 2013;123(7):3154-3165.

5. Smith E, Lin C, Shilatifard A. The super elongation complex (SEC) and MLL in development and disease. Genes Dev. 2011;25(7):661-672.

6. Yu BD, Hess JL, Horning SE, Brown GA, Korsmeyer SJ. Altered Hox expression and segmental identity in Mll-mutant mice. Nature. 1995;378(6556):505-508.

7. McMahon KA, et al. Mll has a critical role in fetal and adult hematopoietic stem cell self-renewal. Cell Stem Cell. 2007;1(3):338-345.

8. Krivtsov AV, Armstrong SA. MLL translocations, histone modifications and leukaemia stem-cell development. Nat Rev Cancer. 2007;7(11):823-833.

9. Super HJ, et al. Rearrangements of the MLL gene in therapy-related acute myeloid leukemia in patients previously treated with agents targeting DNAtopoisomerase II. Blood. 1993;82(12):3705-3711.

10. Liedtke M, Cleary ML. Therapeutic targeting of MLL. Blood. 2009;113(24):6061-6068.

11. Thorgeirsson SS. The central role of the c-Met pathway in rebuilding the liver. Gut. 2012; 
61(8):1105-1106

12. Huh CG, Factor VM, Sanchez A, Uchida K, Conner EA, Thorgeirsson SS. Hepatocyte growth factor/ c-met signaling pathway is required for efficient liver regeneration and repair. Proc Natl Acad Sci US A. 2004;101(13):4477-4482.

13. Ishikawa $\mathrm{T}$, et al. Hepatocyte growth factor/ c-met signaling is required for stem-cell-mediated liver regeneration in mice. Hepatology. 2012; 55(4):1215-1226.

14. Trusolino L, Bertotti A, Comoglio PM. MET signalling: principles and functions in development, organ regeneration and cancer. Nat Rev Mol Cell Biol. 2010;11(12):834-848

15. Gomez-Quiroz LE, Factor VM, Kaposi-Novak $\mathrm{P}$, Coulouarn C, Conner EA, Thorgeirsson SS Hepatocyte-specific c-Met deletion disrupts redox homeostasis and sensitizes to Fas-mediated apoptosis. J Biol Chem. 2008;283(21):14581-14589.

16. Marquardt JU, et al. Loss of c-Met accelerates development of liver fibrosis in response to
$\mathrm{CCl}(4)$ exposure through deregulation of multiple molecular pathways. Biochim Biophys Acta. 2012; 1822(6):942-951

17. Boccaccio C, Comoglio PM. Invasive growth: a MET-driven genetic programme for cancer and stem cells. Nat Rev Cancer. 2006;6(8):637-645.

18. Kaposi-Novak P, Lee JS, Gomez-Quiroz L, Coulouarn C, Factor VM, Thorgeirsson SS. Metregulated expression signature defines a subset of human hepatocellular carcinomas with poor prognosis and aggressive phenotype. J Clin Invest. 2006;116(6):1582-1595.

19. Santoro A, et al. Tivantinib for second-line treatment of advanced hepatocellular carcinoma: a randomised, placebo-controlled phase 2 study. Lancet Oncol. 2013;14(1):55-63.

20. Santoro A, et al. A Phase-1b study of tivantinib (ARQ 197) in adult patients with hepatocellular carcinoma and cirrhosis. Br J Cancer. 2013; 108(1):21-24

21. Takeda S, et al. Proteolysis of MLL family proteins is essential for taspase 1-orchestrated cell cycle progression. Genes Dev. 2006;20(17):2397-2409.

22. Schmidt C, et al. Scatter factor/hepatocyte growth factor is essential for liver development. Nature. 1995;373(6516):699-702.

23. Fujimoto A, et al. Whole-genome sequencing of liver cancers identifies etiological influences on mutation patterns and recurrent mutations in chromatin regulators. Nat Genet. 2012;44(7):760-764.

24. Linardou $\mathrm{H}$, et al. Assessment of somatic k-RAS mutations as a mechanism associated with resistance to EGFR-targeted agents: a systematic review and meta-analysis of studies in advanced nonsmall-cell lung cancer and metastatic colorectal cancer. Lancet Oncol. 2008;9(10):962-972.

25. Marquardt JU, Andersen JB. Next-generation sequencing: application in liver cancer-past, present and future? Biology. 2012;1(2):383-394.

26. Uchino K, et al. Hepatocellular carcinoma with extrahepatic metastasis: clinical features and prognostic factors. Cancer. 2011;117(19):4475-4483.

\title{
Mutation signature of adenoid cystic carcinoma: evidence for transcriptional and epigenetic
} reprogramming

\author{
Henry F. Frierson Jr. and Christopher A. Moskaluk \\ University of Virginia Health Sciences Center, Charlottesville, Virginia, USA.
}

\begin{abstract}
Adenoid cystic carcinoma (ACC), a relatively rare malignancy usually of salivary gland origin, has a signature v-myb avian myeloblastosis viral oncogene homolog-nuclear factor I/B (MYB-NFIB) gene fusion that activates MYB transcriptional regulatory activity. A new study in this issue by Stephens et al. is a comprehensive genomic mutation profiling analysis of this neoplasm and documents a common theme of alteration in chromatin regulatory genes. Also, mutations in SPEN (split ends, homolog of Drosophila), which encodes an RNA-binding coregulatory protein, suggest that other changes in transcriptional regulation may involve the NOTCH, FGFR, or other signaling pathways in which SPEN participates. Since there is a low prevalence of mutations in common oncogenes and tumor-suppressor genes, it is likely that alterations primarily in specific transcriptional regulatory genes, augmented by changes in chromatin structure, drive the neoplastic process in ACC.
\end{abstract}

In this issue of JCI, Stephens et al. (1) report the results of exome sequencing of 24 cases of adenoid cystic carcinoma (ACC), a relatively rare tumor, but one that is among the most common malignancies arising in salivary glands. ACC has distinctive clinical and pathologic features, including an often lengthy clinical course before the majority of patients succumb to their disease (2), a proclivity for tumor cells to

Conflict of interest: The authors have declared that no conflict of interest exists.

Citation for this article: J Clin Invest. 2013; 123(7):2783-2785. doi:10.1172/JCI69070. invade nerves, which may lead to incomplete surgical resection and recurrence, and distinct myoepithelial/luminal epithelial cellular differentiation. The elucidation of the specific molecular events that underlie ACC may lead to targeted metastases for whom there currently are no effective chemotherapeutic agents.

\section{MYB-NFIB is the signature molecular alteration}

The study by Stephens et al. (1) confirms the presence of activation of $\mathrm{v}$-myb avian myeloblastosis viral oncogene homolog (MYB) therapies for patients who have distant (on chromosome 6) in the majority of ACC (19/24, 79\%); this occurs chiefly by chromosomal translocation and fusion to nuclear factor I/B (NFIB) (on chromosome 9). This key oncogenic event, first discovered in 2009 by Persson et al. (3) in Goran Stenman's laboratory, appears to result in increased concentration and activity of the MYB transcriptional regulatory protein domains. The overexpression of MYB, which may be dysregulated by other mechanisms in ACC that lack MYB-NFIB fusion (4), leads to altered expression of its putative target genes involved in cell-cycle control, apoptosis, cell growth, angiogenesis, and cell adhesion (3). Which of these genes is the most critical for the growth and maintenance of ACC remains to be proven experimentally.

Aside from MYB alterations, Stephens et al. (1) report a mean of 13 mutations per exome in ACC, a mutation rate lower than that reported in comprehensive sequencing analyses of the most common types of carcinoma. The relative stability of the ACC genome at the nucleotide level is in keeping with comparative genomic hybridization (CGH) and array CGH studies that have revealed relatively few copy number alterations per genome 